\title{
First test of a partial Siberian snake during polarized beam acceleration
}

\author{
B. B. Blinov ${ }^{(a)}$, C. M. Chu, E. D. Courant ${ }^{(b)}$, D. A. Crandell, W. A. Kaufman, A. D. Krisch, \\ T. S. Nurushev, R.A. Phelps, D. B. Raczkowski, L. G. Ratner ${ }^{(b)}$, and V. K. Wong ${ }^{(c)}$ \\ Randall Laboratory of Physics, University of Michigan, Ann Arbor, Michigan 48109-1120 \\ D. D. Caussyn ${ }^{(d)}$, Ya. S. Derbenev ${ }^{(e)}$, T. J. P. Ellison, S. Y. Lee, T. Rinckel, P. Schwandt, \\ F. Sperisen, E. J. Stephenson and B. von Przewoski \\ Indiana University Cyclotron Facility, Bloomington, Indiana 47408-0768 \\ R. Baiod ${ }^{(1)}$, M. G. Minty ${ }^{(2)}$, C. Ohmori ${ }^{(3)}$, U. Wienands ${ }^{(4)(f)}$ \\ (1) Fermilab, Batavia, Mlinois 60510 \\ (2) Stanford Linear Accelerator Center, Stanford, California \\ (3) Institute for Nuclear Study, University of Tokyo, Tanashi, Tokyo 188, JAPAN \\ (4) SSC Lab, Dallas, Texas \\ (Received 21 June 1994)
}

\begin{abstract}
We recently studied the first acceleration of a spin-polarized beam through a depolarizing resonance using a partial Siberian snake. We accelerated polarized protons from 95 to $140 \mathrm{MeV}$ while ramping a $10 \%$ partial Siberian snake along with the acceleration cycle. The $10 \%$ partial snake suppressed all observable depolarization due to the $G \gamma=2$ imperfection depolarizing resonance which occurred near $108 \mathrm{MeV}$ during acceleration. However, $20 \%$ and $30 \%$ partial Siberian snakes apparently moved the $G \gamma=7-\nu_{y}$ intrinsic depolarizing resonance from near $177 \mathrm{MeV}$ into our energy range; this caused some interesting but not-yet-fully understood depolarization.
\end{abstract}

PACS Numbers: 41.75 AK, 07.77.+p, 29.27.Bd, 29.27. $\mathrm{Hj}$

Many spin-depolarizing resonances occur during the acceleration of a polarized proton beam in a high energy circular accelerator. The individual resonance correction technique used at the ZGS [1], Saturne [2], KEK [3], and the AGS [4] becomes impractical above about $20 \mathrm{GeV}$. Recent experiments at the IUCF Cooler Ring [5-8] suggested that a full Siberian snake [9] should simultaneously overcome all depolarizing resonances. A full Siberian snake forces all depolarizing effects to cancel themselves by rotating each proton's spin by $180^{\circ}$ on each pass through the snake. This spin rotation can be produced by using either a solenoid with a longitudinal magnetic field, or several transverse dipole magnets, which distort the beam orbit only inside the snake itself. A transverse Siberian snake is especially effective at high energies, while a longitudinal solenoid snake works best at low energies.

The orbit distortion problem [10], caused by a transverse Siberian snake near injection, is especially serious in medium energy accelerators such as the Fermilab $8 \mathrm{GeV}$ Booster [11], and the Brookhaven AGS [12]. In such accelerators, partial Siberian snakes might overcome the many imperfection depolarizing resonances, while modest pulsed quadrupoles could jump through the few weak intrinsic depolarizing resonances.

We studied a partial Siberian snake's ability to overcome depolarizing resonances during beam acceleration, 
by placing two rampable warm solenoid magnets symmetrically around our existing superconducting solenoid. This combination of three magnets allowed us to maintain a fixed partial Siberian snake strength of $10 \%, 20 \%$ or $30 \%$ during acceleration; we could then study a partial snake's ability to overcome the $G \gamma=2$ imperfection depolarizing resonance at $108 \mathrm{MeV}$ during acceleration.

With no Siberian snake, each proton's spin precesses around the ring's vertical magnetic field; however, any horizontal magnetic fields can depolarize the beam. This depolarization occurs when the spin precession frequency, $f_{s}$, satisfies the depolarizing resonance condition

$$
f_{s}=f_{c} \nu_{s}=f_{c}\left(n+m \nu_{y}\right)
$$

where $n$ and $m$ are integers; $f_{c}$ is the protons' circulation frequency; the vertical betatron tune, $\nu_{y}$, is the number of vertical betatron oscillations during each turn around the ring; and the spin tune, $\nu_{s}$, is the number of spin precessions during each turn around the ring. The imperfection resonances occur when $m=0$, while the first-order intrinsic resonances occur when $\mathrm{m}= \pm 1$.

During acceleration with no Siberian snake, the spin tune is proportional to the proton's energy

$$
\nu_{s}=G \gamma
$$

where $\gamma$ is the Lorentz energy factor and $G=1.792847$ is the proton's anomalous magnetic moment. A recent experiment [7] confirmed that, in a ring containing a partial Siberian snake of strength $s$, the spin tune obeys the equation

$$
\cos \left(\pi \nu_{s}\right)=\cos (\pi G \gamma) \cos \left(\frac{\pi s}{2}\right)
$$

where $s=1$ corresponds to a full snake, which rotates the spin by $180^{\circ}$. Note that for a full Siberian snake, the spin tune is equal to a half-integer at all energies.

To create a Siberian snake of variable $\int B \cdot d \ell$, we recently built two rampable warm $0.2 \mathrm{~T} \cdot \mathrm{m}$ solenoids of 1026 turns each. These warm solenoids then bracketed our $2 \mathrm{~T} \cdot \mathrm{m}$ superconducting solenoid as shown in Fig. 1. The superconducting solenoid, the polarimeter and the Cooler Ring's operation with polarized protons were discussed earlier $[5-8,13-15]$. The snake strength, $s$, for a solenoid magnet of $N I$ ampere turns is given by

$$
s=\frac{\mu_{0}(1+G)}{10.479 p} N I,
$$

where $\mu_{0}=4 \pi 10^{-7} \mathrm{~T} \cdot \mathrm{m} \mathrm{A} \mathrm{A}^{-1}$ and $p$ is the proton's momentum in $\mathrm{GeV} / \mathrm{c}$. At $8.4 \mathrm{~A}$, the superconducting solenoid was about a $6.2 \%$ snake at $95 \mathrm{MeV}$, according to the earlier calibration [15].

The polarized proton beam was then injected into the Cooler Ring at $95 \mathrm{MeV}$, which is well below the $G \gamma=2$ imperfection depolarizing resonance [5] near $108 \mathrm{MeV}$. The beam was then accelerated to $140 \mathrm{MeV}$, which is well 
above the $G \gamma=2$ resonance, but still below $177 \mathrm{MeV}$, which is the normal energy of the $G \gamma=7-\nu_{y}$ intrinsic depolarizing resonance [6].

We first studied the depolarization during this acceleration from 95 to $140 \mathrm{MeV}$ with the Siberian snake turned off. In a second study, the three solenoids were run together to maintain a $10 \%$ partial Siberian snake during the acceleration from 95 to $140 \mathrm{MeV}$. The superconducting solenoid current was held fixed at $8.4 \mathrm{~A}$; thus during this acceleration it decreased from being about an $6.2 \%$ snake at $95 \mathrm{MeV}$ to about a $5 \%$ snake at $140 \mathrm{MeV}$. The two warm solenoid magnets were varied together from about $24 \mathrm{~A}$ to about $39 \mathrm{~A}$ each. This variation kept the total snake strength of the three solenoids fixed at about $10 \%$ during acceleration from 95 to $140 \mathrm{MeV}$. In each study we measured the beam polarization for different values of the imperfection $\int B \cdot d \ell$, which was produced by the correction solenoid magnets in the Ring's cooling section shown in Fig. 1.

The transverse beam polarization, $P_{t}=\sqrt{P_{v}^{2}+P_{r}^{2}}$, after acceleration from 95 to $140 \mathrm{MeV}$, is plotted against the imperfection $\int B \cdot d \ell$ in Fig. $2[16]$. Note that the spin direction was flipped both with and without the partial Siberian snake. With a $0 \%$ snake, there is a significant decrease in $P_{t}$ for non-zero $\int B \cdot d \ell$ due to the $G \gamma=2 \mathrm{im}$ perfection depolarizing resonance. Since the beam accelerated through the resonance with $\langle d \gamma / d t\rangle=0.061 \mathrm{~s}^{-1}$, this $P_{t}$ curve is flatter than the $104 \mathrm{MeV}$ fixed-energy data [5]; nevertheless, with no snake, the $G \gamma=2$ resonance clearly depolarized the accelerated beam or rotated its spin into the unmeasurable longitudinal direction.

However, with a $10 \%$ partial Siberian snake, the beam polarization measured after acceleration to $140 \mathrm{MeV}$ was almost independent of the imperfection $\int B \cdot d \ell$ within our precision of about $2 \%$. Note that there may be a slight negative slope to the data; the dashed line is a constant polarization fit to these $10 \%$ snake data. These $0 \%$ and $10 \%$ data clearly demonstrate that a weak partial Siberian snake can overcome an imperfection depolarizing resonance during acceleration.

We also studied the effect of stronger partial Siberian snakes. In Fig. 3, the transverse beam polarization [16], $P_{t}=\sqrt{P_{v}^{2}+P_{r}^{2}}$, after acceleration from 95 to $140 \mathrm{MeV}$ is plotted against the imperfection $\int B \cdot d \ell$ for partial Siberian snakes of $0 \%, 10 \%, 20 \%$, and $30 \%$. Note the unexpected behavior in the $20 \%$ and $30 \%$ snake data; the polarization rapidly decreases at higher $\int B \cdot d \ell$; the change is especially sharp in the $20 \%$ data. This depolarization probably occured because the stronger partial snakes moved the $G \gamma=7-\nu_{y}$ intrinsic depolarizing resonance into our energy region. The partial Siberian snake probably shifted the spin tune according to Eq. 3; this shift moved the intrinsic resonance energy. Moreover, the snake's strong solenoid focusing probably shifted the vertical betatron tune, $\nu_{y}$, which also moved the intrinsic resonance energy. The Cooler solenoids, which created the imperfection $\int B \cdot d \ell$, could also move the intrinsic

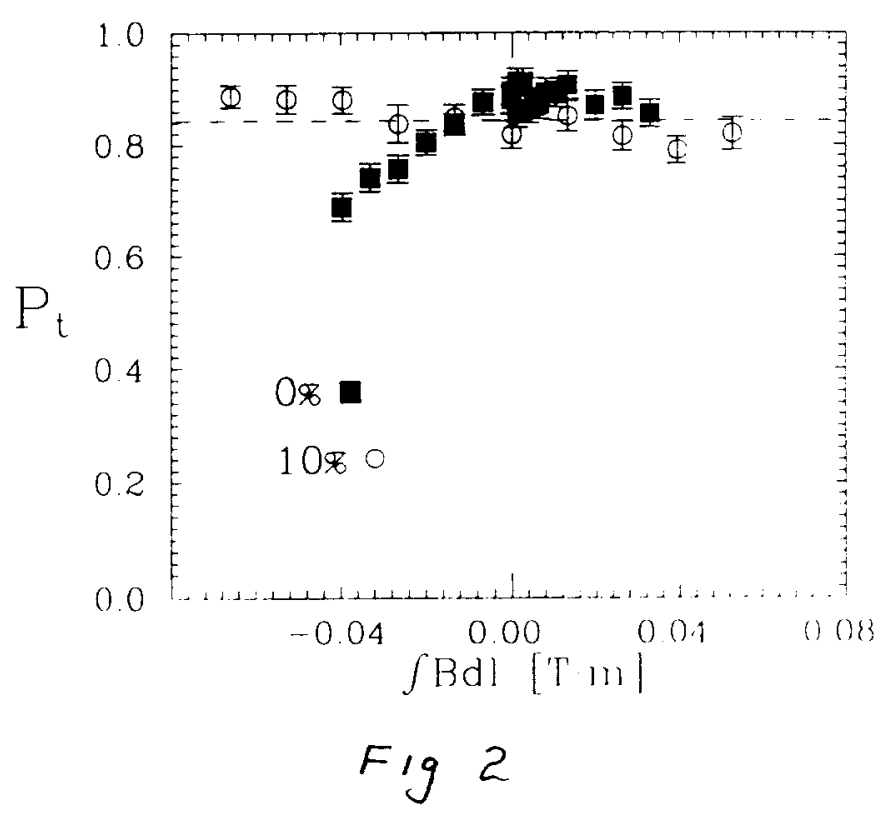


resonance by shifting $\nu_{s}$ and $\nu_{y}$ [17]. All these shifts may have caused the unexpected polarization drop in the $20 \%$ and $30 \%$ data of Fig. 3; however, we do not yet have a detailed explanation for this behavior. Note that the $20 \%$ data drops very sharply.

Thus, the $G \gamma=7-\nu_{y}$ intrinsic depolarizing resonance, which normally occurs near $177 \mathrm{MeV}$ in the Cooler Ring, is apparently shifted by a $20 \%$ or $30 \%$ partial Siberian snake into our 95 to $140 \mathrm{MeV}$ energy range. However, with a $10 \%$ partial snake, this intrinsic resonance apparently stays well outside the 95 to $140 \mathrm{MeV}$ acceleration range; the $10 \%$ snake easily overcame the $G \gamma=2$ imperfection resonance as shown in Fig. 2.

To summarize, the $10 \%$ snake data support the conjecture that a weak partial Siberian snake can maintain full beam polarization during acceleration through a weak imperfection depolarizing resonance. Therefore, partial Siberian snakes might allow the acceleration of a polarized proton beam at medium-energy accelerators such as the Fermilab $8 \mathrm{GeV}$ Booster [11] or the Brookhaven AGS [12] by simultaneously using modest pulsed quadrupoles to jump quickly through the few weak intrinsic resonances. However, the apparently complex behavior of partial Siberian snakes, near intrinsic depolarizing resonances, highlights the need to further study their interaction.

We would like to thank J. M. Cameron and the entire Indiana University Cyclotron Facility staff for the most successful operation of the Cooler Ring. We are grateful to V. A. Anferov, A. W. Chao, S. V. Gladysheva, R. S. Herdman, S. Hiramatsu, F. Z. Khiari, A. V. Koulsha, W. F. Lehrer, H.-O. Meyer, J. B. Muldavin, R. E. Pollock, T. Roser, H. Sato, D. S. Shoumkin, S. E. Sund, T. Toyama and S. J. Wheeler for their help with parts of this experiment. This research was supported by grants from the U.S. Department of Energy and the U.S. National Science Foundation.

(a) Also at: Moscow State University, Moscow, Russia.

(b) Also at: Brookhaven National Laboratory.

(c) Also at: Office of the Provost, University of Michigan at Flint.

(d) Now at: Department of Physics, University of Michigan.

(e) Also at: Department of Nuclear Engineering, University of Michigan.

(f) Now at: Stanford Linear Accelerator Center.

[1] T. Khoe et al., Part. Accel. 6, 213 (1975).

[2] J. L. Laclare et al., J. Phys. (Paris), Colloq. 46, C2-499 (1985).

[3] H. Sato et al., Nucl. Instrum. Methods Phys. Res. Sect. A 272, 617 (1988).

[4] F. Z. Khiari et al., Phys. Rev. D39, 45 (1989).

[5] A. D. Krisch et al., Phys. Rev. Lett. 63, 1137 (1989).

[6] J. E. Goodwin et al., Phys. Rev. Lett. 64, 2779 (1990).

[7] V. A. Anferov et al., Phys. Rev. A46, R7383 (1992).

[8] R. Baiod et al., Phys. Rev. Lett. 70, 2557 (1993).

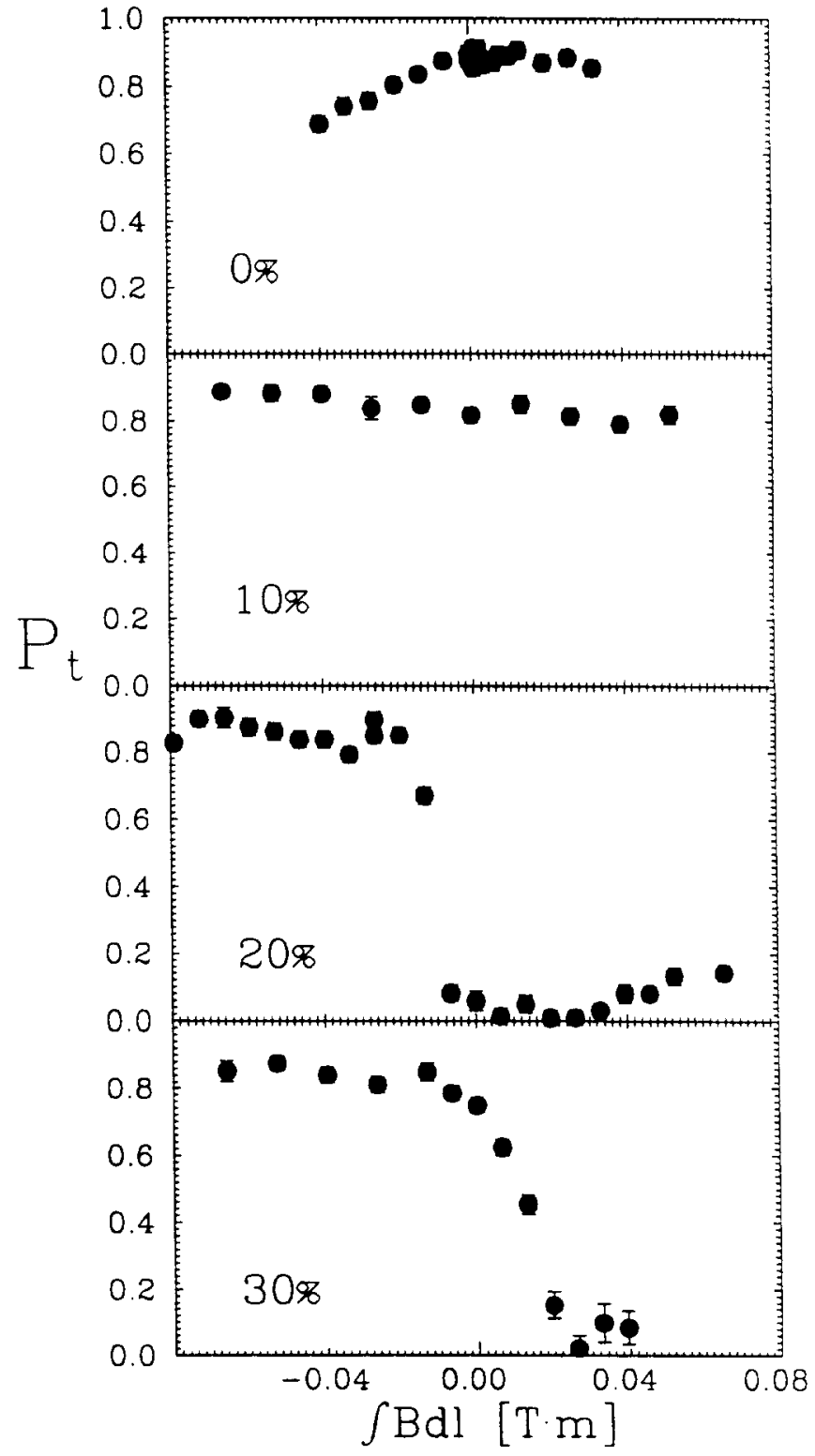

FIG 3 
[9] Ya. S. Derbenev and A. M. Kondratenko, Part. Accel. 8, 115 (1978)

[10] Proceed. 1985 Ann Arbor Workshop on Polarized Beams at $S S C$, AIP Conf. Proc. 145, eds. A. D. Krisch, A. M. T. Lin, O. Chamberlain (AIP, New York 1986).

[11] Acceleration of Polarized Protons to 120 and $150 \mathrm{GeV}$ in the Fermilab Main Injector, SPIN Collaboration, Univ. of Michigan Report (March 1992), (unpublished).

[12] RHIC Spin Proposal M. Beddo et al.; Brookhaven National Lab proposal (1992), (unpublished).

[13] R. A. Phelps et al., Phys. Rev. Lett. 72, 1479 (1994).

[14] M. G. Minty et al., Phys. Rev. D44, R1361 (1991).

[15] J. E. Goodwin, Ph.D. Thesis, Indiana University (1990).

[16] In Figs 2 and 3, there is a normalization uncertainty of about $15 \%$ which fortunately does not effect the shape of the curves. This first attempt to interpolate to $140 \mathrm{MeV}$ data at nearby energies clearly gave too low an $\mathrm{A}$ in our polarimeter's angular range. Certainly the Cooler Ring polarization can not be larger than the injected polarization which was about $75 \%$.

[17] The vertical betatron tune $\nu_{y}$, measured by the IUCF staff, was typically constant within \pm 0.01 during acceleration. Even this small $\Delta \nu_{y}=0.01$ shifts the intrinsic resonance energy by about $5.2 \mathrm{MeV}$.

FIG. 1. Location of the two warm rampable solenoids, the superconducting solenoid, and other relevant hardware in the IUCF Cooler Ring

FIG. 2. The measured [16] transverse polarization, $P_{t}=\sqrt{P_{v}^{2}+P_{r}^{2}}$, at $140 \mathrm{MeV}$ is plotted against the imperfection $\int B \cdot d \ell$ with no snake and with a $10 \%$ partial Siberian snake. The dashed line is the best constant polarization fit to the snake-on data. The beam was accelerated from 95 to 140 $\mathrm{MeV}$.

FIG. 3. The measured [16] transverse polarization, $P_{t}=\sqrt{P_{v}^{2}+P_{r}^{2}}$, at $140 \mathrm{MeV}$ is plotted against the imperfection $\int B \cdot d \ell$ for a partial Siberian snake of strength $0 \%$, $10 \%, 20 \%$ and $30 \%$. The beam was accelerated from 95 to $140 \mathrm{MeV}$. 
